\title{
Benefícios da atividade física para a promoção da saúde dos idosos com Alzheimer: uma revisão de literatura
}

Benefits of physical activity for health promotion of elderly with Alzheimer: a literature review

\author{
Michele Duarte da Silva Xavier Xavier \\ Universidade Federal do Rio de Janeiro \\ michelefreitasxavier@hotmail.com \\ Leona Rabelo Santos Barros \\ UGB/FERP \\ leona-barros@hotmail.com

\section{Leandro Oliveira} \\ Universidade Federal do Rio de Janeiro \\ Itoliveira@hotmail.com

\section{Camilli Fernanda Martins dos Santos}

Universidade Federal do Rio de Janeiro camilli.fernanda.m@gmail.com

\section{Diego Viana-Gomes}

Universidade Federal do Rio de Janeiro

diegoefd@gmail.com

\section{Rodrigo Peixoto dos Santos}

UGB/FERP

peixotofisio@yahoo.com.br 


\section{RESUMO}

A incidência da doença de Alzheimer (DA), forma mais comum de demência em idosos, tem aumentado à medida que a população mundial envelhece, com milhões de pessoas afetadas em todo o mundo. Até o momento não existe um tratamento eficaz para DA, e esforços significativos são direcionados no sentido de desenvolver novas estratégias, como atividade física regular, para neutralizar os mecanismos que levam a danos neuronais. Neste sentido, este estudo teve como objetivo identificar os efeitos que a atividade física proporciona para o tratamento de idosos com Alzheimer. A metodologia aqui empregada foi uma revisão literária através de livros e artigos. Com base nessas observações, apresentamos que a atividade física regular pode auxiliar na prevenção e no tratamento da DA, sendo praticado pelo paciente e pelos cuidadores; cabendo aos profissionais de Educação Física, o aprofundamento cada vez maior em estudos destas bases, além de informar sobre os benefícios da atividade física para o auxílio do tratamento dessa doença.

Palavras-Chave: Atividade Física, Envelhecimento, Alzheimer, Neurodegeneração

\section{ABSTRACT}

Physical activity is a tool for health promotion. The incidence of Alzheimer's disease (AD), the most common form of dementia in the elderly, has increased as the world's population ages, with millions of people affected worldwide. To date, there is no effective treatment for $A D$, and significant efforts are directed towards developing new strategies to counteract the mechanisms that lead to neuronal damage. In this sense, this study aimed to identify the beneficial effects that physical activity provides for the treatment of elderly people with Alzheimer's. The methodology used here was a literary review through books and articles. Based on these observations, we present here how physical activity can help in the prevention and treatment of $A D$, being practiced by the patient and caregivers; It is up to Physical Education professionals to deepen further studies on these bases, in addition to inform about the benefits of physical activity to aid in the treatment of this disease.

Keywords: Physical activity, Aging, Alzheimer's, Neurodegeneration 


\section{INTRODUÇÃO}

A Doença de Alzheimer (DA) se caracteriza por uma patologia neurodegenerativa sem cura responsável por mais de $50 \%$ das doenças relacionadas à demência, seu predomínio cresce exponencialmente com a idade (Ritchie et al, 2017). Uma variedade de potenciais mecanismos foram propostos para explicar a patogênese da DA (Lourenço, Ferreira e De Felice, 2015). Dentre eles, está associada ao acúmulo de placas amilóides extraneuronais e emaranhados neurofibrilares intraneuronais, sendo subdividida em três fases, sendo elas: leve, moderada e grave, seguida da deterioração de outras funções cognitivas de acordo com o avanço da doença.

Conforme apontado por Sereniki \& Vital (2008), a DA já é a terceira causa de morte nos países desenvolvidos, ficando apenas atrás do câncer e das doenças cardiovasculares. No censo realizado pelo IBGE, em 2010 , os idosos brasileiros já totalizavam $11 \%$ da população e nas próximas décadas, em 2030, estima-se que já serão $13,4 \%$ da população (Martins, Caldas, Cabral, Lins e Coriolano, 2019).

0 processo natural do envelhecimento relaciona-se com a redução de algumas capacidades físicas que podem levar à incapacidade funcional (Colón-Emeric, Whitson, Pavon e Hoenig, 2013), as quais podem ser atenuadas com exercícios regulares. A prática regular de atividade física é aspecto fundamental na promoção da saúde de idosos e na prevenção de doenças relacionadas ao envelhecimento, pois ajuda no desenvolvimento cognitivo e psicomotor do paciente com o potencial de reduzir ou retardar a progressão dessas doenças assim como contribuir para sua reabilitação sendo apontado por induzir eventos relacionados à memória no cérebro (Van Praag Fleshner, Schwartz e Mattson, 2014).

Ainda não existe intervenção médica no sentido de prevenção e já foram observados fatores de risco associados ao desenvolvimento de demência relacionado ao estilo de vida dos indivíduos e dentre eles o baixo nível de atividade física (De La Rosa et al, 2020). Sendo assim, tanto mulheres quanto homens idosos necessitam do exercício físico para garantir resultados não somente relacionados ao retardamento do declínio das funções cognitivas, assim como, na mobilidade dos músculos e das articulações, na prevenção de quedas, no fortalecimento de ossos e músculos desta forma promovendo um envelhecimento mais saudável de forma global (Orlando, Silva e Lombardi Junior, 2013).

Neste sentido organizações como a American College of Sports Medicine (2003) formularam diretrizes para a prática de atividade física para este público, recomendam o exercício aeróbio para o idoso em intensidade moderada, com uma frequência semanal de três dias, duração mínima de 20 minutos e intensidade variando entre 55 a $85 \%$ da frequência cardíaca máxima (FCmax.) dependendo do nível de condicionamento do 
indivíduo e salienta a participação de idosos em atividades físicas regulares de exercícios aeróbios e de força de forma a contribuir para o envelhecimento saudável.

0 objetivo geral do estudo foi compreender e demonstrar efeitos benéficos da atividade física na recuperação da plasticidade sináptica e formação de memória, para promoção da saúde dos idosos acometidos pelo Alzheimer com exercícios sistematizados, que possam colaborar com o bem-estar e habilidades físicas dessas pessoas, apresentandose assim como uma promissora ferramenta potencial para intervenção terapêutica na DA.

\section{MÉTODOS}

Este estudo tem o carácter descritivo e exploratório. Foram analisadas as pesquisas publicadas até maio de 2021, nas bases de dados PubMed, Scielo e Lilacs. As palavraschave que orientaram a escolha da revisão foram: atividade física, idosos e Alzheimer. A triagem dos artigos se baseou nos estudos relacionados aos objetivos e critérios de inclusão e exclusão citados a seguir.

Critérios de inclusão: estudos longitudinais, abertos (grupo experimental) e de coorte; estudos randomizados e não randomizados; amostras constituídas por indivíduos acima de 60 anos e com diagnóstico clínico de DA; e estudos contendo intervenção com exercício físico e atividade cognitiva.

Critérios de exclusão: estudos que não envolviam intervenção por meio de exercícios físicos; e aqueles em que a amostra não era exclusivamente composta por idosos com DA, mas incluía idosos com outras demências ou declínio cognitivo leve.

\section{RESULTADOS E DISCUSSÃO}

A participação em atividade física regular (exercícios aeróbicos e de força) fornece respostas favoráveis que contribuem para o envelhecimento saudável. Houve um aumento dos conhecimentos recentemente em relação à adaptabilidade do sistema biológico assim como os meios com os quais o exercício regular pode influenciá-lo.

Com o objetivo de verificar a relação entre exercícios regulares e a saúde, Civinski, Montibeller e De Oliveira (2011), concluíram que o declínio nos níveis de atividade física habitual para o idoso contribui de maneira significativa para a redução da aptidão funcional e a manifestação de diversas doenças relacionadas a este processo, trazendo como 
consequência a perda da capacidade funcional. Neste sentido, tem sido enfatizada a prática de exercícios físicos como estratégia para prevenir as perdas nos componentes da aptidão física e funcional da saúde desta população.

Segundo Da Silveira et al (2011) o treinamento aeróbico, aplicado a um grupo de idosos, influenciou significativamente na autonomia e na qualidade de vida desta população. Conforme esse estudo, as atividades praticadas e mais recomendadas para os idosos são as atividades aeróbias de baixa intensidade, associadas a exercícios com pesos para estimular a manutenção da força muscular dos membros superiores e inferiores (Matsudo, Matsudo e Neto 2001).

A prescrição de exercícios para o idoso é desafiante porque há muitas questões envolvidas, entre elas as clínicas e psicológicas. Dessa forma se faz necessária uma avaliação geriátrica abrangente que contemple todos os aspectos inseridos no envelhecimento. Coelho et al, 2010, apontam melhora significativa em idosos com doenças neurodegenerativas. A atividade física contribui com o desenvolvimento cognitivo e psicomotor do paciente, com o potencial de reduzir ou retardar a progressão da doença e assim contribuindo para sua reabilitação. Pesquisas têm demonstrado a ação benéfica do exercício físico no quadro dessas doenças, como um tratamento não farmacológico, por exemplo, no Alzheimer resultando em efeitos positivos na cognição, nos distúrbios de comportamento e melhora na função motora de pacientes com DA (Arcoverde et al, 2008).

A atividade física é de fundamental relevância para a terceira idade, promovendo mais saúde de diferentes maneiras, tais como: melhorando a agilidade, promovendo novas amizades, aumentando a coordenação motora, reduzindo o cansaço e a solidão, aumentando a resistência, a flexibilidade e o fortalecimento muscular. Ou seja, a prática regular de saúde não só evita a doença, mas também permite maior bem-estar físico, mental e social (OMS). Gonçalves, (2003) descreve que a função muscular possui ampla relevância na autonomia do idoso, porque as atividades diárias requerem força muscular. 0 treinamento de força destaca-se como um instrumento de grande valia nesta independência por aprimorar o aumento de força e massa muscular.

Assim como descreve Cardoso (2012, p. 09-10), "a prática de atividades físicas, esportivas e recreativas é essencial na vida das pessoas, porquanto além de ser importante como veículo de educação para a promoção da saúde, compõe uma realização expressiva de lazer". Em relação à prevenção de doenças, o treino de força muscular deve ser indicado em casos de aumento do peso corporal, artrite grave, desordens do equilíbrio, ulcerações no pé, amputação, doença pulmonar e baixo limiar para isquemia. Assim, a musculação é um treinamento que pode parar ou reverter à perda de massa muscular e adequar à manutenção da capacidade funcional e independência (Matsudo, Matsudo e Barros Neto, 2001). Além disso, condicionando a prática de atividade física 
uma extrema importância para o auxílio do tratamento de doenças neurodegenerativas, pois ajuda no desenvolvimento cognitivo e psicomotor do paciente com o potencial de reduzir ou retardar a progressão da doença e assim contribuindo para sua reabilitação como comentado anteriormente (Arcoverde et al, 2008).

\section{CONCLUSÃO}

A DA é um distúrbio neurológico que afeta principalmente a memória e, até o presente momento, sem cura. Uma variedade de potenciais mecanismos foi proposta para explicar a patogênese da DA (Lourenço el al. 2015; Katsnelson et al, 2016). 0 exercício físico já foi apontado por induzir eventos relacionados à memória no cérebro (Van Praag $\mathrm{H}$ et al, 2014; Neufer et al, 2015; Suwabe et al, 2018) e proposto como uma abordagem para reduzir o risco de DA, podendo trazer benefícios significativos para indivíduos com DA precoce ou comprometimentos cognitivos leves (Lourenço et al, 2019).

Nosso estudo apresenta evidências de numerosos benefícios que a atividade física pode oferecer à saúde de pessoas de todas as idades e a sua importância, sobretudo, na terceira idade em que a declínio das funções cognitivas além da diminuição de outras funções. A prática de exercício físico, com ênfase no treinamento de força, visto que a atividade de musculação ajuda na prevenção de diabetes, doenças cardiovasculares, impede demência, entre outras que prejudicam a qualidade de vida do idoso. A atividade física e/ou treinamento de força promove maior autonomia ao idoso, isto é, ele alcança independência funcional e qualidade de vida.

Assim, é importante que mais estudos e pesquisas, como os que estimulam à prática da atividade física direcionada a população idosa, tenham continuação objetivando comprovar os numerosos benefícios contraídos com esses exercícios, podendo assim apresentar uma nova estratégia terapêutica para proteger/reparar os danos sinápticos e prevenir o declínio cognitivo na DA. 


\section{BIBLIOGRAFIA}

Arcoverde, C., Deslandes A., Rangel A., Rangel A., Pavão R., Nigri F., Engelhardt E., Laks J. (Jun, 2008). Role of physical activity on the maintenance of cognition and activities of daily living in elderly with Alzheimer's disease. Arq Neuropsiquiatr; 66 (2B): pp. 323-7.

Traduzido por: Lazzoli J. K. (1990) A quantidade e o tipo recomendados de exercícios para o desenvolvimento e a manutenção da aptidão cardiorrespiratória e muscular em adultos saudáveis (The recommended amount and type of exercise for the development and maintenance of cardiorespiratory and muscular fitness in healthy adults. Med Sci Sports Exerc; 22: 265-74). (Mai/Jun, 1998). Revista Brasileira de Medicina Do Esporte, 4(3), pp. 96-106.

Cardoso, J. R. (jun 2012). Atividades físicas para a terceira idade (Physical activities for seniors). Revista A Terceira Idade, São Paulo, SP, v.4, n.4, pp. 9-10.

Carvalho, E. B. \& Neri, A. L. (2018). Time use by family caregivers of elderly with dementia: an integrative review. Revista Brasileira de Enfermagem, 71(suppl 2), pp. 893-904.

Civinski, C.; Montibeller, A.; De oliveira, AL (2011). A importância do exercício físico no envelhecimento (The importance of physical exercise in aging.). Revista da UNIFEBE, v. 1, n. 09.

Coelho, F. H. N.; Natalli, B. V. A. (Sep. 2010). Benefícios da musculação na terceira idade (Benefits of weight training for seniors). Revista Digital, Buenos Aires, 15, n. 148.

Colón-Emeric C. S., Whitson H. E., Pavon J., Hoenig H. (2013 Sep). Functional decline in older adults. Am Fam Physician. 15;88(6):388-94. PMID: 24134046; PMCID: PMC3955056.

De la Rosa A, Olaso-Gonzalez G., Arc-Chagnaud C., Millan F., Salvador-Pascual A., GarcíaLucerga C., Blasco-Lafarga C., Garcia-Dominguez E., Carretero A., Correas AG, Viña J., Gomez-Cabrera M. C. (2020 Feb 4). Physical exercise in the prevention and treatment of Alzheimer's disease. J Sport Health Sci. 2020 Sep;9(5): pp.394-404.

Da Silveira, M. M., Kümpel, D. A., Rocha, J. P, Wibelinger, L. M., Pasqualotti, A., \& Colussi, E. L. (Jan-Mar 2013). Atividade física e doenças crônicas em adultos e idosos (Physical activity and chronic diseases in adults and elderly). Revista Contexto \&Amp; Saúde, 11(20), pp. 695-700. 
Gonçalves, V. (Acesso: 17 jun. 2006). Exercícios de forca para idosos. Maio, 2003. Disponível em: <www.educacaofisica.com.br/mostra_biblioteca.asp?>

Katsnelson A., De Strooper B., Zoghbi H. Y. Neurodegeneration: From cellular concepts to clinical applications. Sci Transl Med. (2016 Nov) 9;8(364):364ps18.

Kamada, M., Clemente J. S., Monteiro A. F. F., Barros L. V. G., Helene A. H. E., Morato D. M. (2018). Correlação entre exercício físico e qualidade de vida em pacientes com doença de Alzheimer (Correlation of physical exercise and quality of life in patients with Alzheimer's disease). Revista da Sociedade Brasileira de Clínica Médica, v. 16, n. 2, pp. 119-122.

Lourenco, M. V., Ferreira S. T., De Felice F. G. (2015, Jun). Neuronal stress signaling and elF2 $\alpha$ phosphorylation as molecular links between Alzheimer's disease and diabetes. Prog Neurobiol.;129:37-57.

Matsudo, S. M.; Matsudo, V. K. R.; Neto B. T. L. (Jan-Fev. 2001). Atividade física e envelhecimento: aspectos epidemiológicos (Physical activity and aging: epidemiological aspects). Revista Brasileira de Medicina do Esporte, v. 7, n. 1, pp. $2-13$.

Martins, N. I. M., Caldas P. R., Cabral E. D., Lins C. C. D. S. A., Coriolano M.D.G.W.S (2019 Jul). Cognitive assessment instruments used in elderly Brazilians in the last five years. Cien Saude Colet. 22;24(7):2513-2530. Portuguese, English.

Monteiro, W. D. (1996). Aspectos fisiológicos e metodológicos do condicionamento físico na promoção da saúde (Physiological and methodological aspects of physical conditioning in health promotion). Revista Brasileira de Atividade Física \& Saúde, v. 1, n. 3, pp. 44-58.

Oliveira, D. V., Pivetta R. N. S., Oliveira G. V. N., Silva D. A., Nascimento Júnior J. R. A., Cavaglieri C. R. (2019). Fatores intervenientes nos indicativos de depressão em idosos usuários das unidades básicas de saúde de Maringá, Paraná, 2017 (Intervening factors in the indicators of depression in elderly users of basic health units in Maringá, Paraná, 2017). Epidemiologia e Serviços de Saúde, v. 28, p. e2018043, 2019.Pe

Orlando, M. M., Silva, M. S. P. \& Lombardi Junior, I. (2013). The influence of the practice of physical activity on the quality of life, muscle strength, balance, and physical ability in the elderly. Revista Brasileira de Geriatria e Gerontologia, 16(1), pp. 117126.

Ritchie, C., Smailagic N., Noel-Storr A. H., Ukoumunne O., Ladds E., Martin S. (2017 Mar). CSF tau and the CSF tau/ABeta ratio for the diagnosis of Alzheimer's disease 
dementia and other dementias in people with mild cognitive impairment ( $\mathrm{MCl})$. Cochrane Database Syst Rev. 22;3(3):CD010803.

Santiago, A. M., Souza E, Maldonado A, Rodrigues M, Leme J. A. C.A. (2016). Efeitos da participação em programa de atividade física para pessoas com a Doença de Alzheimer (Effects of participation in a physical activity program for people with Alzheimer's disease.). Fisioterapia Brasil, v. 17, n. 3, pp. 261- 268.

Sereniki, A, \& Vital, M. A. B. F. (2008). A doença de Alzheimer: aspectos fisiopatológicos e farmacológicos (Alzheimer's disease: pathophysiological and pharmacological features). Revista de Psiquiatria Do Rio Grande Do Sul, 30(1 suppl),.

Siqueira, J. F., Antunes M. D., Nascimento Junior J. R. A., Oliveira D. C. (2019). Efeitos da prática de exercício de dupla tarefa em idosos com Doença de Alzheimer: revisão sistemática (Effects of dual-task exercise in elderly people with Alzheimer's disease: a systematic review.). Saúde e Pesquisa, v. 12, n. 1, pp. 197-202.

Suwabe, K., Byun K., Hyodo K., Reagh Z. M., Roberts J. M., Matsushita A ., ... Soya H.( 2018). Rapid stimulation of human dentate gyrus function with acute mild exercise. Proceedings of the National Academy of Sciences 115, pp. 10487-10492.

Van Praag, H., Fleshner M., Schwartz M. W., Mattson M. P. (Nov 2014). Exercise, energy intake, glucose homeostasis, and the brain. Journal of Neuroscience 34, pp. 1513915149 .

Neufer, P. D., Bamman M. M, Muoio D. M, Bouchard C, Cooper D. M. ... Laughlin M. R. (2015 Jul). Understanding the Cellular and Molecular Mechanisms of Physical Activity-Induced Health Benefits. Cell Metab. 7;22(1):pp. 4-11.. 